\title{
Catalytic Asymmetric Functionalization of Inert Bonds and Synthesis of Bioactive Natural Products
}

\author{
Nicolai Cramer
}

\begin{abstract}
The direct and enantioselective functionalization of inert bonds such as carbon-hydrogen and carboncarbon is an emerging tool towards more sustainable and efficient synthetic methods. The individual activation pathways like concerted deprotonation metalations, directed activations, $\beta$-carbon eliminations or retro-allylations proceed by completely different mechanisms and therefore have complementary requirements and different associated challenges. A careful fine-tuning of the transition-metal complex is critical for each mechanism, but a very broad structural space can be covered as well. These methods enhance the synthetic chemist's toolbox allowing more concise, efficient synthetic routes to be executed in target-oriented synthesis. This is illustrated by the examples of a synthesis of largazole and the core of stachyflin from our group.
\end{abstract}

Keywords: Asymmetric catalysis · C-C activation $\cdot \mathrm{C}-\mathrm{H}$ activation $\cdot$ Natural products $\cdot$ Transition metals

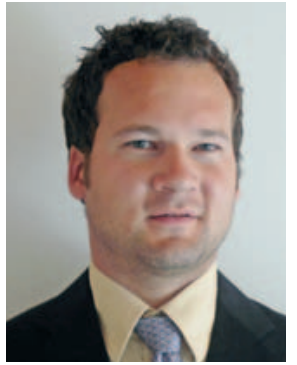

\section{Introduction}

Since the foundation of my laboratory, first at the ETH Zurich and since fall 2010 at the EPF Lausanne, the main research objective of my group has spanned two areas: In the first, we aim to design and develop synthetic methods basing on novel and selective transitionmetal catalyzed activation modes that are of broad applicability and synthetic versatility. The second major topic comprises the synthesis and synthetic modification of structurally challenging natural products that possess intriguing biological activities.

\section{Catalytic Functionalizations of Inert Bonds}

Transition metal-catalyzed transformations have become a very powerful and indispensable tool enabling reactions that would be otherwise impossible. Most principal reaction types, especially those for $\mathrm{C}-\mathrm{C}$ bond formations, need functionalized substrates, usually in the form of carbonhalide $(\mathrm{C}-\mathrm{X})$ and carbon-main group metal $(\mathrm{C}-\mathrm{M})$ bonds. To circumvent these prefunctionalization requirements, the direct use of unactivated carbon-hydrogen bonds in catalysis has received increasing attention in recent years. New reaction protocols have been reported that furnish important building blocks in a previously unknown fashion (Scheme 1). [1] However, general and reliable approaches are still lacking and often the transformations require fairly high catalyst loadings, high temperatures and harsh conditions which are not compatible with several of the most useful functional groups.

The broad implications and the potential of concerted deprotonation metalation (CMD) pathways for the functionalization of $\mathrm{C}-\mathrm{H}$ bonds have been more and more recognized over the past years and the mechanistic understanding and reaction protocols have been refined. ${ }^{[2]}$ However, a chiral version was lacking and one has to design and develop completely new ligand systems that could enable such reactivity. Whereas we have recently successfully showed the proof of principle for aromatic $\mathrm{C}\left(\mathrm{sp}^{2}\right)-\mathrm{H}$ bonds, the corresponding catalytic enantioselective activation of saturated $\mathrm{C}\left(\mathrm{sp}^{3}\right)-\mathrm{H}$ remains a significant and so far unmet synthetic challenge. Using an engineered TADDOL-based phosphoramidite ligand (L1), it is not only possible to promote a highly enantioselective activation process of $\mathbf{1}$, but the reaction occurs as well under extraordinarily mild conditions at ambient temperature in the presence of sodium bicarbonate (Scheme 2). ${ }^{[3]}$ With this important initial report, we are encouraged to generalize this reactivity to render $\mathrm{C}-\mathrm{H}$ bonds of further substrate classes available for related enantioselective functionalizations.

Nitrogen-directing groups like 2-pyridyl or oxazolyl groups have evolved in the last decade as a very fruitful pathway for directed $\mathrm{C}-\mathrm{H}$ bond functionalizations. The major drawback of this approach is that the directing group is usually neither
*Correspondence: Prof. N. Cramer

Ecole polytechnique fédérale de Lausanne EPFL SB ISIC LCSA $\mathrm{BCH} 4305$

$\mathrm{CH}-1015$ Lausanne

Tel.: +41216939839

E-mail: nicolai.cramer@epfl.ch

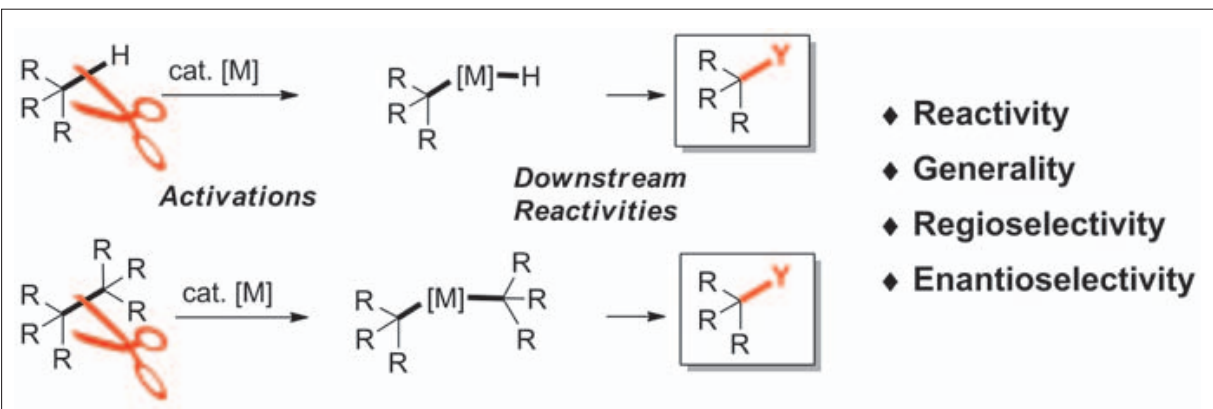

Scheme 1. Synthetic potential by $\mathrm{C}-\mathrm{H}$ and $\mathrm{C}-\mathrm{C}$ bond functionalizations. 


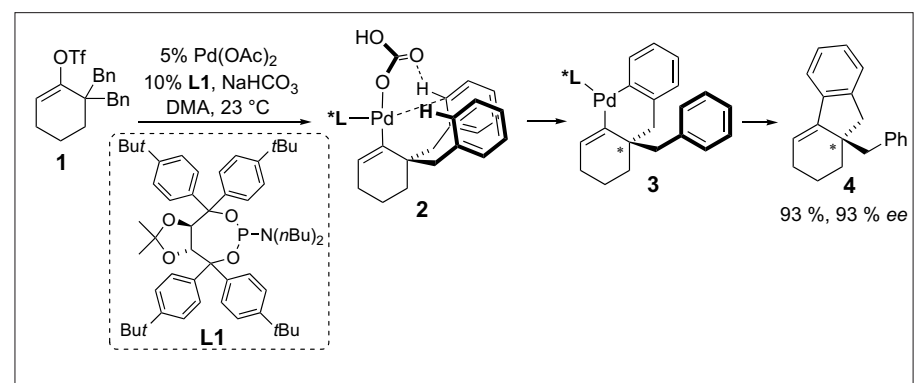

Scheme 2. Example of an enantioselective palladium-

catalyzed $\mathrm{C}\left(\mathrm{sp}^{2}\right)-\mathrm{H}$ functionalization by the CMD pathway operating at ambient temperature.

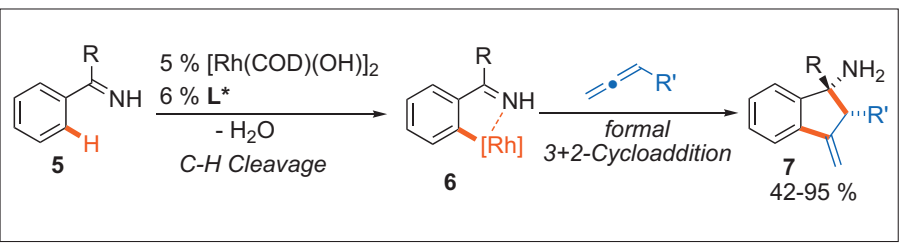

Scheme 3. Rhodium(1)catalyzed nitrogendirected $\mathrm{C}\left(\mathrm{sp}^{2}\right)-\mathrm{H}$ functionalizations in formal $3+2$ cycloadditions with allenes.

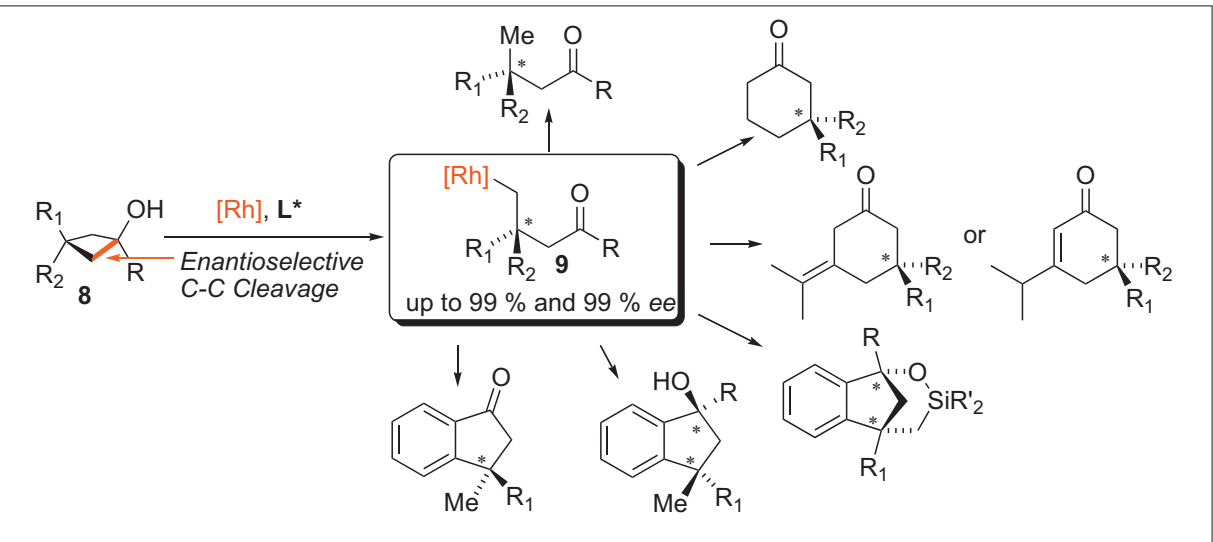

Scheme 4. Rhodium(I)-catalyzed enantioselective $\beta$-carbon eliminations and their different downstream reaction pathways.

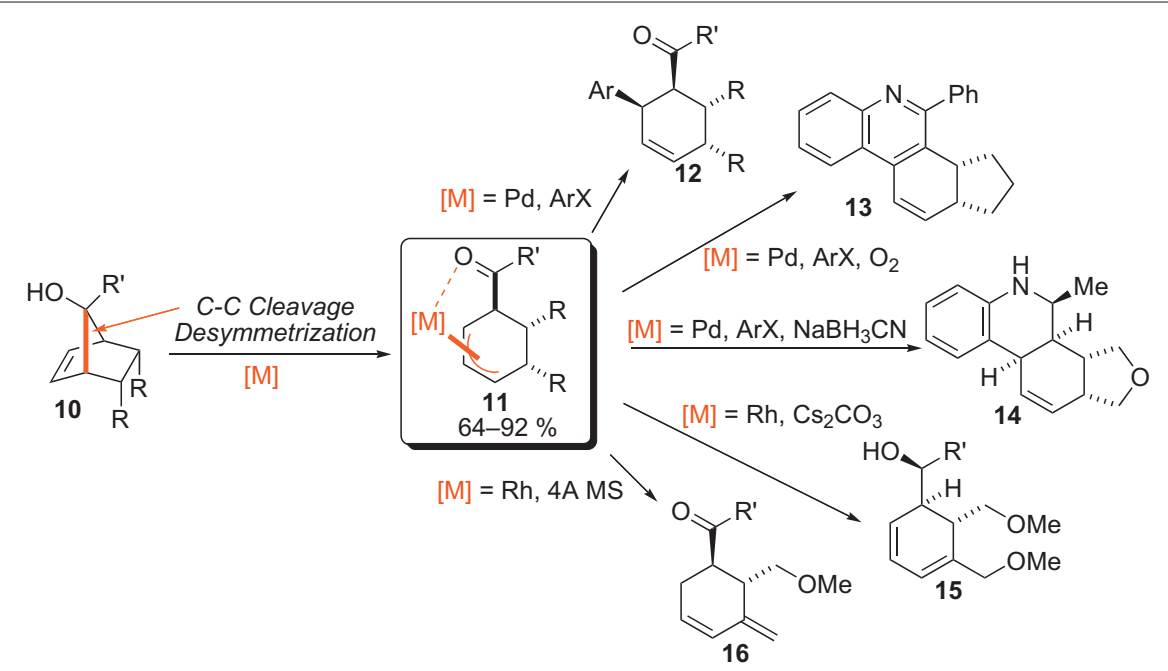

Scheme 5. Synthetic versatility of allyl-metal intermediates generated by retro-allylative $\mathrm{C}-\mathrm{C}$ bond cleavages of homoallylic alcohols.

common nor easily interconvertable in organic synthesis. We seek to identify enabling directing groups that have the benefit to be synthetically attractive and modifiable, or that are convertible 'on the fly' into another valuable functionality during the course of the reaction. A representative example towards this goal is outlined in Scheme 3. As the initial step, unsubstituted ketimines $\mathbf{5}$ are used to direct ortho-aryl $\mathrm{C}-\mathrm{H}$ bond activation with a rhodium(I) complex. In turn, intermediate $\mathbf{6}$ reacts with olefin acceptors such as terminal allenes to give valuable methylene indanylamines 7. From a purely synthetic point of view, this can be regarded as a formal 3+2-cycloaddition. ${ }^{[4]}$ A deeper insight of this process was discussed within the framework of a SCS DSM poster award earlier this year in this journal. ${ }^{[5]}$

The inertness of $\mathrm{C}-\mathrm{C} \sigma$-bonds is even more pronounced than for $\mathrm{C}-\mathrm{H}$ bonds as they are more directional and shielded. More favorable reverse pathways like $\mathrm{C}-\mathrm{C}$ bond-forming reductive eliminations from intermediates complicate the process further. As a consequence, the development of catalytic carbon-carbon single bond activations is highly challenging and asymmetric transformations are extremely scarce. In this respect, $\beta$-carbon eliminations from strained tertiary alcohols $\mathbf{8}$ have emerged as a highly versatile tool to access uncommon primary and uniquely reactive alkyl-rhodium intermediates 9 bearing an enantio-enriched quaternary stereogenic center (Scheme 4). ${ }^{6]}$ The different reaction pathways of intermediate 9 depend on the reaction conditions, ligands and substitution pattern and lead to a range of highly diverse products in high yields and enantioselectivities. ${ }^{[7]}$ The different product-forming steps and selectivities have been described already in this journal. ${ }^{[8]}$

Importantly, $\beta$-carbon cleavages are not exclusively limited to strained cyclobutanes but could also be extended to other, less activated, ring systems. For instance, bicyclic tertiary alcohols such as $\mathbf{1 0}$ are desymmetrized by an insertion of a metal complex into a $\mathrm{C}-\mathrm{C}$ bond, providing $\pi$-allyl species 11 (Scheme 5). This intermediate opens numerous possibilities for further downstream reactivities using transition-metal catalysts.

With palladium complexes, highly diastereoselective $\mathrm{C}-\mathrm{C}$ bond formation occurs with aryl halides, yielding cyclohexenes $\mathbf{1 2}$ with four contiguous stereogenic centers. ${ }^{[9]}$ No diastereo- or regioselective scrambling is observed. The concomitantly formed carbonyl moiety of $\mathbf{1 1}$ can be utilized as well for high complexity increasing domino reactions. In this respect, $o$-bromo aniline coupling partners yield under oxidative conditions with air quinolines $\mathbf{1 3}$ and provide under reductive conditions with sodium cyano borohydride selectively tetrahydroquinolines $\mathbf{1 4}$. In stark contrast, complementary pathways are dominant performing the corresponding reaction with rhodium(I) complexes. For instance, with a cesium carbonate additive, secondary alcohol $\mathbf{1 5}$ is formed selectivity, whereas in the presence of $4 \AA$ molecular sieves, diene $\mathbf{1 6}$ dominates. ${ }^{[10]}$ This is an insightful example of how shallow energy profiles between different reaction pathways allow the reaction outcome to be altered by carefully adjusting the reaction conditions. 


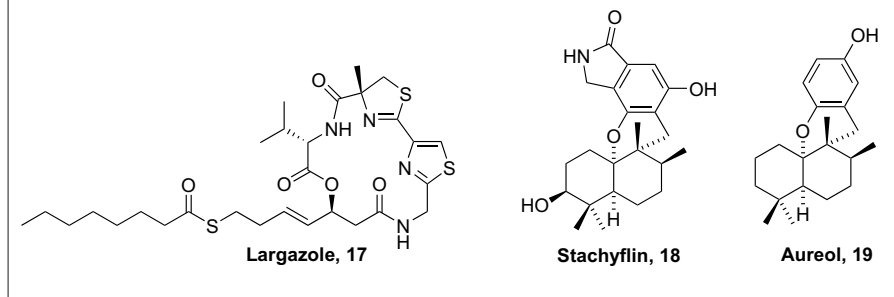

Fig.1. Examples of natural products which interesting bioactivities and synthetic challenges.

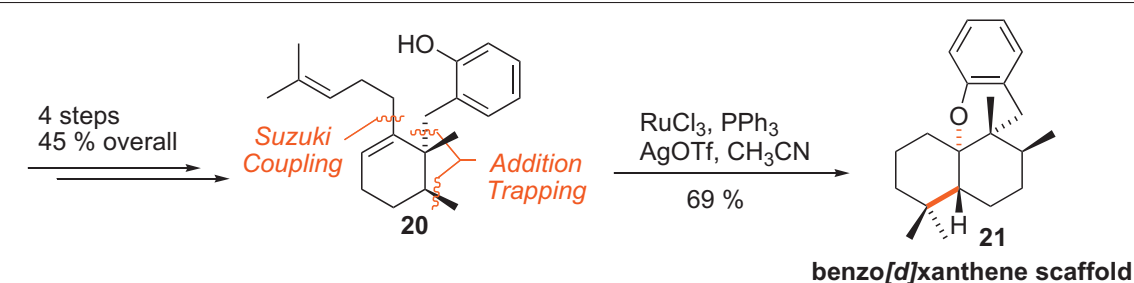

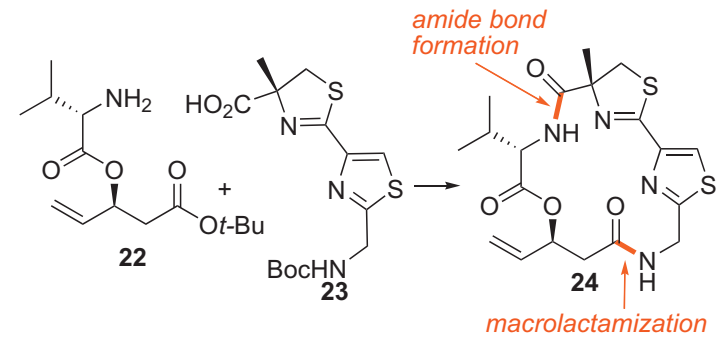

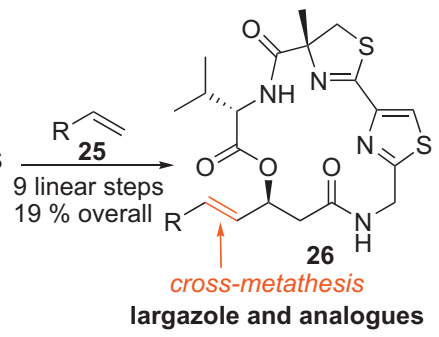

Scheme 6. Transition-metal catalysis as key strategy in efficient synthetic routes.

showed that these reactions provide new opportunities for a rapid increase in molecular complexity from simple starting materials and have the potential to enhance synthetic efficiency. The focus is shifting now from initial trailblazing prototype reactivity towards the development of transformations that allow access to synthetically valuable structures and building blocks. Although significant progress in the mechanistic understanding and the development of more practical reaction protocols has been made over past years, the substrate scope and the generality of the concepts have to be further increased before the non-specialist will make regularly use of this emerging technology. So far, this has been a joyful ride with a myriad of intriguing discoveries along for us.

\section{Acknowledgments}

This work was supported by the ETH Zurich (ETH-16 08-3), the Swiss National Foundation (21-119750.01) and a Liebig-Fellowship from the Fonds der Chemischen Industrie. Solvias AG, Takasago International Corporation, Umicore AG \& Co. KG and Johnson Matthey are acknowledged for donations of ligands and rhodium salts.

Received: May 16, 2011

\section{Synthesis of Bioactive Natural Product Synthesis}

Our second main research interest covers the area of natural product synthesis. We are especially interested in those compounds that possess a synthetic challenge paired with interesting and rewarding biological activities. Our main aim consists in developing modular syntheses that result in a rapid increase in molecular complexity. This allows for a molecular editing of the core scaffold which is of interest to enlighten the activity profile and mode of action of the individual molecules. For example, we are interested in the two natural products, largazole $(\mathbf{1 7})^{[11]}$ and stachyflin (18) ${ }^{[12]}$ (Fig. 1). Both have been isolated in only minute quantities from their producing organisms.

Stachyflin (18) and aureol (19) are members of a class of sesquiterpenes with a benzo $[d]$ xanthene scaffold and are produced by fungi and/or marine sponges. Among other biological activities, especially stachyflin exhibits a potent antiinfluenza activity which caught our interest in this class of compounds. We have set our goal to establish a robust synthetic platform that should enable an access to the core structure itself and furthermore provide a wide range of analogues. Towards these goals, we have developed a concise five-step synthesis to the benzo[d] xanthene core $\mathbf{2 1}$ which is prepared in a highly enantioselective manner using three consecutive transition-metal catalyzed processes (Scheme 6). ${ }^{[13]}$

On the other hand, largazole (17) was isolated from cyanobacteria and displays a potent, yet surprisingly selective, antiproliferative activity. This feature and its - at the point of discovery unknown molecular target, prompted us to evaluate its potential closer. Our modular synthesis using the three readily accessible fragments 22, 23 and 25 allows largazole and key analogues $\mathbf{2 6}$ in to be prepared in nine linear synthetic steps and $19 \%$ overall yield (Scheme 6).[14] Different olefins $\mathbf{2 5}$ employed in the crucial crossmetathesis step allowed some SAR data to be established and led to conclusions on the potent histone deacetylase inhibitory activity of largazole. Further routes and analogues have been published by different research groups and provide more insights into the potential of this inhibitor. ${ }^{[15]}$ Although it is pure coincidence that for both natural products ruthenium catalysts are involved to forge key bonds in the molecules, it underlines once again the possibilities of transition-metal catalysis to streamline synthetic routes.

\section{Conclusion}

The outlined catalytic functionalization reactions of the $\mathrm{C}-\mathrm{H}$ and $\mathrm{C}-\mathrm{C}$ bonds
[1] 'C-H Activation', Eds. J.-Q. Yu, Z. Shi, Top. Curr. Chem. 2010, 292.

[2] S. I. Gorelsky, D. Lapointe, K. Fagnou, J. Am. Chem. Soc. 2008, 130, 10848.

[3] M. R. Albicker, N. Cramer, Angew. Chem. Int. Ed. 2009, 48, 9139.

[4] D. N. Tran, N. Cramer, Angew. Chem. Int. Ed. 2010, 49, 8181 .

[5] D. N. Tran, N. Cramer, Chimia 2011, 65, 271.

[6] a) T. Seiser, N. Cramer, Org. Biomol. Chem. 2009, 7, 2835; b) N. Cramer, T. Seiser, Synlett 2011, 449 .

[7] a) T. Seiser, N. Cramer, Angew. Chem. Int. Ed. 2008, 47, 9294; b) T. Seiser, O. A. Roth, N. Cramer, Angew. Chem. Int. Ed. 2009, 48, 6320; c) T. Seiser, N. Cramer, Chem. Eur. J. 2010, 16, 3383; d) T. Seiser, N. Cramer, J. Am. Chem. Soc. 2010, 132, 5340; e) T. Seiser, G. Cathomen, N. Cramer, Synlett 2010, 1699; f) T. Seiser, N. Cramer, Angew. Chem. Int. Ed. 2010, 49, 10163.

[8] T. Seiser, N. Cramer, Chimia 2010, 64, 153.

[9] M. Waibel, N. Cramer, Angew. Chem. Int. Ed. 2010, $49,4455$.

[10] M. Waibel, N. Cramer, Chem. Commun. 2011 , $47,346$.

[11] K. Taori, V. J. Paul, H. Luesch, J. Am. Chem. Soc. 2008, 130, 1806

[12] K. Minagawa, S. Kouzuki, J. Yoshimito, Y. Kawamura, H. Tani, T. Iwata, Y. Terui, H. Nakai, S. Yagi, N. Hattori, T. Fujiwara, T. Kamigauchi, J. Antibiot. 2002, 55, 155.

[13] D. T. Ngoc, M. Albicker, L. Schneider, N. Cramer, Org. Biomol. Chem. 2010, 8, 1781.

[14] T. Seiser, F. Kamena, N. Cramer, Angew. Chem. Int. $E d . \mathbf{2 0 0 8}, 47,6483$.

[15] a) T. Seiser, N. Cramer, Chimia 2009, 63, 19; b) T. L. Newkirk, A. A. Bowersab, R. M. Williams, Nat. Prod. Rep. 2009, 26, 1293. 